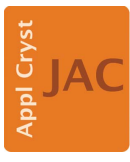

JOURNAL OF

APPLIED

CRYSTALLOGRAPHY

ISSN 1600-5767

\section{A Rietveld refinement method for angular- and wavelength-dispersive neutron time-of-flight powder diffraction data}

\author{
Philipp Jacobs, ${ }^{a}$ Andreas Houben, ${ }^{a}$ Werner Schweika, ${ }^{\text {b,c }}$ Andrei L. Tchougréeff ${ }^{\mathrm{a}, \mathrm{d}}$ \\ and Richard Dronskowski ${ }^{\mathrm{a} *}$
}

Received 24 June 2015

Accepted 4 September 2015

Edited by D. Pandey, Indian Institute of Technology (Banaras Hindu University), Varanasi, India

Keywords: Rietveld refinement; neutron diffraction; angular-dispersive; wavelength-dispersive; powder methods; VITESS; POWTEX; POWGEN

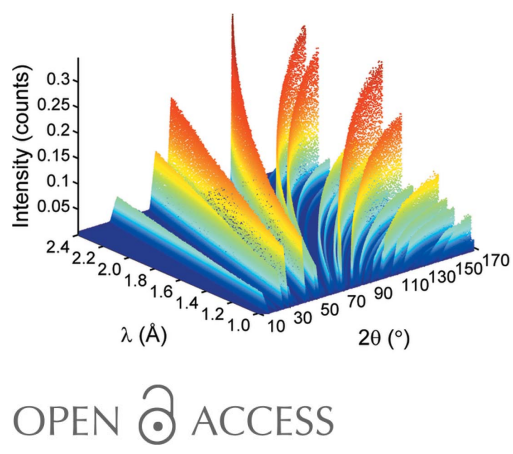

${ }^{a}$ Chair of Solid-State and Quantum Chemistry, Institute of Inorganic Chemistry, RWTH Aachen University, Aachen, D-52056, Germany, 'buropean Spallation Source ESS, Lund, SE-22100, Sweden, 'Jülich Center for Neutron Science and Peter Grünberg Institute PGI, Forschungszentrum Jülich, Jülich, D-52425, Germany, and ${ }^{\mathbf{d}}$ Department of Chemistry, and Poncelet Laboratory of Mathematics in Interaction with Physics and Informatics, Lomonosov Moscow State University, Independent University of Moscow, Moscow Center for Continuous Mathematical Education, Bolshoi Vlasevsky Per. 11, Moscow, 119002, Russian Federation. *Correspondence e-mail: drons@HAL9000.ac.rwth-aachen.de

This paper introduces a two-dimensional extension of the well established Rietveld refinement method for modeling neutron time-of-flight powder diffraction data. The novel approach takes into account the variation of two parameters, diffraction angle $2 \theta$ and wavelength $\lambda$, to optimally adapt to the varying resolution function in diffraction experiments. By doing so, the refinement against angular- and wavelength-dispersive data gets rid of common data-reduction steps and also avoids the loss of high-resolution information typically introduced by integration. In a case study using a numerically simulated diffraction pattern of $\mathrm{Rh}_{0.81} \mathrm{Fe}_{3.19} \mathrm{~N}$ taking into account the layout of the future POWTEX instrument, the profile function as parameterized in $2 \theta$ and $\lambda$ is extracted. As a proof-of-concept, the resulting instrument parameterization is then utilized to perform a typical refinement of the angular- and wavelengthdispersive diffraction pattern of $\mathrm{CuNCN}$, yielding excellent residuals within feasible computational efforts. Another proof-of-concept is carried out by applying the same approach to a real neutron diffraction data set of CuNCN obtained from the POWGEN instrument at the Spallation Neutron Source in Oak Ridge. The paper highlights the general importance of the novel approach for data analysis at neutron time-of-flight diffractometers and its possible inclusion within existing Rietveld software packages.

\section{Introduction}

Since the early days of the ingenious Rietveld method (Rietveld, 1969) in the 1960s, the method has become widely applied because it allows crystallographic and, using neutron data, even magnetic structure investigations on powdered, polycrystalline samples. The slowly but steadily evolving computational power in the 1970s and the proper description of the individual peak shapes were two essential steps for moving away from the simple interpretation of total integrated intensities towards a least-square fit of the full diffraction profile. This holds especially true for overlapping peaks as encountered in low-symmetry structures or in multiphase samples. As a presumably lucky coincidence, the neutron powder diffractometer used by Rietveld appeared to deliver Gaussian-like shapes for the Bragg peaks, just like in the case of modern monochromatic neutron diffractometers. Even though the peak shapes of time-of-flight (TOF) data are certainly more complicated (Von Dreele et al., 1982), the Rietveld method was soon applied for TOF instruments as well. 
When trying to properly describe the peak shape in general, one immediately realizes that a plethora of different effects contribute to the way it looks. The first aim is to separate the instrumental and geometrical effects, e.g. the asymmetry caused by the umbrella effect (Finger et al., 1994; van Laar \& Yelon, 1984) or the change of the peak widths (FWHM, full width at half-maximum) with the scattering variable by changing instrumental resolution, from the intrinsically more interesting sample effects (e.g. crystal structure, particle size, microstrain or texture effects). In current Rietveld algorithms, there are numerous approaches to model the influences of all such effects on the peak shape and intensities (Avdeev et al., 2007; Dollase, 1986; Lutterotti et al., 1999; March, 1932; Popa, 1998; Rodriguez-Carvajal, 1997; Stephens, 1999). One example is the incorporation of classical texture analysis into the Rietveld method as done by the MAUD software (Lutterotti et al., 1999; http://maud.radiographema.com/). Here, the intensity is described as a function of the scattering angle $2 \theta$ and the polar angle $\varphi$ along each Debye-Scherrer cone. Hence, the data acquisition has to account for both variables, which is normally performed using a (one-dimensional) position-sensitive detector to save measurement time or, even better, by using two-dimensional detectors which, in addition, reveal potentially sharp textures.

Modern powder diffractometers (Chapon et al., 2011; Huq et al., 2011; Kamiyama et al., 1995; Peters et al., 2006) at advanced neutron spallation sources (Fischer, 1997; Ikeda, 2005; Lengeler, 1998; Lisowski \& Schoenberg, 2006; Mason et al., 2006) typically operate in TOF mode and use large area detectors, thereby generating angular- and wavelengthdispersive data. This is in contrast to classical monochromatic instruments (Fischer et al., 2000; Garlea et al., 2010; Hansen et al., 2008; Hoelzel et al., 2012; Liss et al., 2006; Többens et al., 2001) at continuous reactor sources as well as typical X-ray powder diffractometers. Current approaches at existing instruments therefore first reduce, transform and integrate the accumulated data to obtain the well known one-dimensional diffraction patterns (MANTID; http://www.mantidproject.org; Schäfer et al., 1992) (e.g. intensity as a function of $2 \theta$ or TOF) that can be routinely treated using the standard software packages (Bruker, 2005; Larson \& Von Dreele, 1994; Lutterotti et al., 1999; Petrríček et al., 2006; Rodríguez-Carvajal, 1993, 1997). For example, the inevitable $\varphi$ dependence encountered at two-dimensional detectors is integrated for each reflection to allow for standard Rietveld refinements. This is normally done by straightening the measured Debye-Scherrer cones detected as circles on flat area detectors (Elf et al., 2002). Although this simple procedure has the advantage of refining diffraction data that are relatively small in size and leads to quick calculations, a significant amount of the available information is lost and cannot be exploited.

In a sense, the present situation resembles the challenges Hugo Rietveld had to meet back in the 1960s: a lack of both computing power and powerful algorithms resulted in an unsatisfying data representation. Indeed, this very datamassaging problem is known to the entire community, and it has also been said: 'One day in the not too far distant future one may leave it curved and introduce the necessary peak shape/resolution functions into a two-dimensional Rietveld refinement' (Kuhs \& Klein, 2008).

Here we shall introduce a novel data-treatment approach for angular- and wavelength-dispersive data sets based on simulation results for the evolving TOF powder diffractometer POWTEX (Conrad et al., 2008; Houben et al., 2012). A few sentences covering the design of that instrument seem in order.

The POWTEX instrument will feature a four-dimensional large area detector (Modzel et al., 2014) covering about 9 steradian of solid angle, and the detection of neutron events will be position sensitive $(x, y, z)$ and time resolved $(t)$, hence four-dimensional. The measured quantities can be readily converted to the Bragg angle $\theta$, the polar angle $\varphi$, the detection depth $z$ and the wavelength $\lambda$. For the present analysis, we will reduce the data by integrating over the polar angle $\varphi$ and the detection depth $z$ so that only the variables $2 \theta$ and $\lambda$ remain. We will lay out all necessary steps, especially how the peak shape is parameterized using both variables and how the varying resolution function is expressed by a suitable parameterization.

Since the Rietveld method represents a least-squares fit that is totally independent of the data dimension, we want to emphasize, however, that one day in the not too distant future one may even apply a Rietveld model refinement to threedimensional and four-dimensional powder diffraction data.

\section{Simulations}

Because of the fact that the POWTEX instrument is currently under construction on beamline SR5a at the FRM II neutron source, real experimental data sets are not available yet. Nonetheless, it is mandatory to (approximately) know the POWTEX data ahead of the instrument's realization, for obvious reasons; this may be accomplished as follows: instrument simulation programs using Monte Carlo methods (Lefmann \& Nielsen, 1999; Lieutenant et al., 2014; Wechsler et al., 2000; Zsigmond et al., 2006) may be used in order to obtain data sets that closely resemble those that will be obtained by the instrument in the future. Consequently, the VITESS program package (Lieutenant et al., 2014; Wechsler et al., 2000; Zsigmond et al., 2006) was utilized to simulate data sets based on instrumental parameters of the POWTEX diffractometer. The main parameters included in the simulation are summarized in Table 1. For simulating the neutron source, a wavelength distribution according to the input file ('FrmII_thermal.dat') of the newest VITESS version for SR5 was used. With respect to the resolution of the real instrument, the simulations account for the neutron-guide definition with its divergence properties, the four-disc chopper system including the double-disc pulse chopper, the sample and the detector geometry. The neutron-guide system was simulated as a polygonal approximation of the (partially) truly curved guide geometry, and it includes the design values of the reflective coating scheme (Houben et al., 2012). The time resolution 
Table 1

Instrumental parameters to generate data sets using the Monte Carlo instrument simulation package VITESS.

\begin{tabular}{ll}
\hline No. starting trajectories & $1.5 \times 10^{12}$ \\
Length of neutron guide No. $1(\mathrm{~m})$ & 27.078 \\
Length of neutron guide No. 2 (m) & 11.096 \\
Double-disc pulse chopper & $\begin{array}{c}\text { Two counter-rotating discs with } 11 / 10 \\
\text { apertures and } 75 \mathrm{~cm} \text { diameter }\end{array}$ \\
Total length chopper to sample (m) & $\begin{array}{l}12.128 \\
\text { Sample }\end{array}$ \\
Detector & Spherical, radius $=1 \mathrm{~cm}$ \\
& Cylindrical, $l=1.6 \mathrm{~m}, r=0.8 \mathrm{~m}$
\end{tabular}

( $\Delta t \simeq 10 \mu \mathrm{s}$ ) is essentially defined by the double-disc pulse chopper.

The sample module determines the scattering of the neutron beam by simply generating a random location along the neutron trajectory through the sample at which the neutron is scattered out of the sample. The scattering process itself is based on structural models given as an input file to the sample module; absorption effects were neglected in these first simulations. The detector system is implemented in monitor mode, meaning that the position of each neutron count is pinpointed to the intersection of the trajectory with the detector surface. For simplicity, the detector's spatial resolution was assumed to be perfect, since the POWTEX's detector characteristics have not yet been defined in all details. The simulations were based on the structural models of $\mathrm{Rh}_{0.81} \mathrm{Fe}_{3.19} \mathrm{~N}$ and $\mathrm{CuNCN}$ (Houben et al., 2005, 2009; Liu et al., 2005; Jacobs et al., 2013) (see Table 2) using a large number of trajectories $\left(1.5 \times 10^{12}\right)$.

After recording all neutron trajectories, the resulting data (position in $x, y, z$; time; trajectory probability, i.e. intensity) were converted to three-variable data sets $(2 \theta, \lambda$, trajectory probability) by applying simple geometrical relations and relating TOF to wavelength. Therefore, a new two-dimensional module called 'eval_elast2' was implemented in VITESS. Herein, the resulting data were integrated over the polar angle $\varphi$ and binned in $2 \theta$ and $\lambda$ using bin sizes of $0.1^{\circ}$ and $0.001 \AA$ (corresponding to approximately a third of the time resolution). The binning mimics a possible sampling grid and is finer than the instrument resolution. Incorporating more instrumental details will be a future task which will not affect the principle of the approach presented.

\section{Neutron powder diffraction}

As a 'real-world' alternative, additional neutron powder diffraction data of $\mathrm{CuNCN}$ were obtained at the POWGEN instrument [Spallation Neutron Source (SNS), Oak Ridge]. The measurement time was approximately $7.5 \mathrm{~h}$. In addition, the background and the vanadium measurements were carried out as well. The Nexus event data of the sample, background and vanadium measurements (stored in the corresponding 'event.nxs' file) were treated according to the standard 'SNSPowderReduction' Python script included in the MantidPlot program package (MANTID). Nonetheless, we omitted the cylindrical absorption correction for the vanadium data and the final diffraction focusing step. Hence, in contrast
Table 2

Crystallographic data of $\mathrm{Rh}_{0.81} \mathrm{Fe}_{3.19} \mathrm{~N}$ and $\mathrm{CuNCN}$.

\begin{tabular}{|c|c|c|}
\hline & $\begin{array}{l}\mathrm{Rh}_{0.81} \mathrm{Fe}_{3.19} \mathrm{~N} \\
\text { (Houben } \text { et al., 2009) }\end{array}$ & $\begin{array}{l}\text { CuNCN } \\
\text { (Jacobs et al., 2013) }\end{array}$ \\
\hline $\begin{array}{l}\text { Lattice parameters } \\
(\AA)\end{array}$ & $a=3.83366(2)$ & $\begin{array}{c}a=2.98908(8), b=6.1420(3) \\
c=9.4009(4)\end{array}$ \\
\hline Space group & $P m \overline{3} m($ No. 212$)$ & $\mathrm{Cmcm}$ (No. 63) \\
\hline Formula units & $Z=1$ & $Z=4$ \\
\hline Atomic sites & $\begin{array}{l}\mathrm{Rh}(1 a) 0|0| 0 \\
\mathrm{~N}(1 b) \frac{1}{2}\left|\frac{1}{2}\right| \frac{1}{2} \\
\mathrm{Fe}(3 c) 0\left|\frac{1}{2}\right| \frac{1}{2}\end{array}$ & $\begin{array}{l}\mathrm{Cu}(4 a) 0|0| 0 \\
\mathrm{C}(4 c) 0|0.3889(8)| \frac{1}{4} \\
\mathrm{~N}(8 f) 0|0.3826(4)| 0.3815(3)\end{array}$ \\
\hline Temperature & $T=300 \mathrm{~K}$ & $T=17 \mathrm{~K}$ \\
\hline
\end{tabular}

to the standard $d$ binning used in MantidPlot (logarithmic binning), in our case the event data are binned in $2 \theta$ as well as in $\lambda$ using bin sizes of $0.1^{\circ}$ and $0.001 \AA$, respectively, matching those for the simulated data on purpose. Subsequently the sample pattern was corrected for background and finally calibrated by the vanadium pattern (from which the vanadium reflections had already been stripped off) to account for detector efficiency and to remove the wavelength-dependent intensity distribution.

\section{Data-analysis approach}

For all calculations presented in this work we used the MATLAB program package (http://www.mathworks.com). All refinements were carried out using functions and code explicitly written (but not yet fully optimized) for the work presented herein.
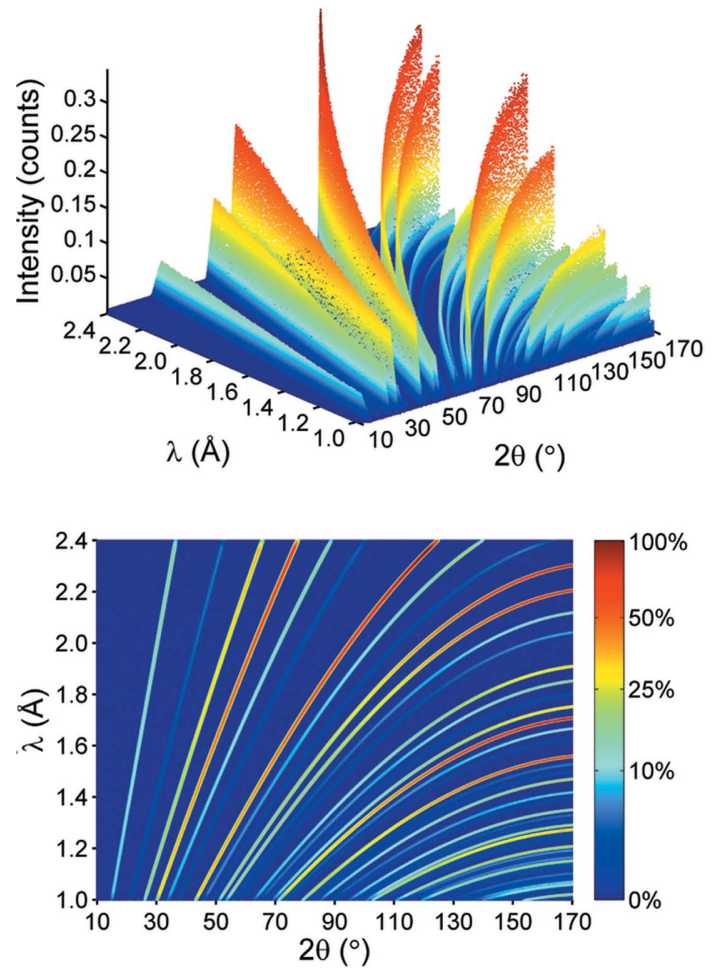

Figure 1

Simulated diffraction pattern $I(2 \theta, \lambda)$ of $\mathrm{Rh}_{0.81} \mathrm{Fe}_{3.19} \mathrm{~N}$ using a twodimensional (top) and quasi-two-dimensional (bottom) representation. 
We reiterate that the simulated data as stored in the output file of the VITESS simulation are intensities as a function of $2 \theta$ and $\lambda$. A simulated pattern for $\mathrm{Rh}_{0.81} \mathrm{Fe}_{3.19} \mathrm{~N}$ is shown in Fig. 1. Although similar patterns are obtainable from other existing TOF instruments with large area detectors, at least in principle, almost no references to two-dimensional diffraction patterns can be found in the literature. Schäfer et al. (1992) provide such a plot but for further analysis the data $I(d)$ were grouped into equally spaced $d$ intervals. This simplification is typical for current approaches at TOF instruments and allows for the use of standard refinement packages.

In Fig. 1, single reflections of constant $d$ spacing are now represented by sinusoidal curves, as given by Bragg's law $(\lambda=2 d \sin \theta)($ Bragg \& Bragg, 1913). Note that the width of each reflection varies with scattering angle and wavelength. In order to perform a Rietveld refinement, i.e. a least-squarestype analysis, one needs to find an analytical description of the diffraction pattern that is able to fit all variable parameters to the measured data set. In the two-dimensional case the calculated intensity $I_{\text {calc }}(2 \theta, \lambda)$ for a single-phase diffraction pattern can be expressed for every data point by

$$
\begin{aligned}
I_{\text {calc }}(2 \theta, \lambda)= & S \sum_{h k l} F_{k h l}^{2} M_{h k l} \operatorname{LAPC}(2 \theta, \lambda) \Omega\left(d_{2 \theta, \lambda}-d_{h k l}\right) \\
& +b(2 \theta, \lambda),
\end{aligned}
$$

with $S$ being a scaling factor, $M_{h k l}$ the multiplicity, $F_{h k l}^{2}$ the structure factor, $\operatorname{LAPC}(2 \theta, \lambda)$ introducing geometrical and physical corrections, $\Omega\left(d_{2 \theta, \lambda}-d_{h k l}\right)$ being the profile function that models both instrumental and sample effects, and $b(2 \theta, \lambda)$ the background. The summation is done over all $h k l$ reflections for each data point. LAPC comprises the Lorentz factor, absorption, preferred orientation and special corrections to the intensity distribution. As the simulation does not include absorption and preferred orientation, these and other such special effects were neglected. The Lorentz factor, on the other hand, has been accounted for and found to be proportional to $d^{4}$ for the simulated data (Von Dreele et al., 1982).

The next step involves finding a suitable description of the profile function for the two-dimensional case. For onedimensional data a lot of effort has been invested over several decades to find good profile functions describing the form and width of the reflection peaks for different instruments and neutron sources (Bacon \& Thewlis, 1949; Cheetham \& Taylor, 1977; Dinnebier \& Billinge, 2008; Young \& Wiles, 1982). A common trait of all current profile functions is that they depend on one variable only (in most cases either $2 \theta$, TOF or $d$ ). In obvious contrast, analyzing two-dimensional data sets will require the reflection profile to be a function of two variables, here wavelength and scattering angle, while the rest of the parameters presented in equation (1) will essentially remain untouched.

Figure 2
For the two-dimensional description of the simulated POWTEX data, an appropriate profile function $\Omega$ needs to be found. Some of the resolution effects that are relevant for the real instrument and contribute to the profile shape are accounted for in the simulations already. The elliptic neutron guide mainly determines the shape of the divergence distribution, which is of particular importance for the profile function $\Omega$, and can be described by a sum of two Gaussians of equal height but shifted by $\pm \delta_{2 \theta, \lambda}$ from the central $d_{h k l}$ value, resulting in a smooth symmetric beam profile. For an arbitrarily chosen data point in the diffraction pattern with $2 \theta=$ $127.8^{\circ}$ and $\lambda=1.988 \AA$, the peak profile $\Omega\left(d_{2 \theta, \lambda}-d_{h k l}\right)$ is shown in Fig. 2. One could easily imagine that the use of different amplitudes, $\sigma$ and $\delta$ values for each of the single Gaussians would even allow one to approximate asymmetric peak shapes; for further information about this topic the reader is referred to Howard (1982). It might indeed become necessary to use a slightly different function for the actually measured POWTEX data but the mathematical procedure described in the following would be very similar.

Note that the profile $\Omega$, the width $\sigma$ and separation $\delta$ of the two Gaussians vary with the diffraction angle $2 \theta$ and the wavelength $\lambda$ :

$$
\begin{aligned}
\Omega\left(d_{2 \theta, \lambda}-d_{h k l}\right)= & \frac{1}{\sigma_{2 \theta, \lambda}}\left(\frac{\ln 2}{\pi}\right)^{1 / 2}\left\{\exp \left[-\frac{\left(d_{2 \theta, \lambda}-d_{h k l}-\delta_{2 \theta, \lambda}\right)^{2}}{\sigma_{2 \theta, \lambda}^{2}}\right]\right. \\
& \left.+\exp \left[-\frac{\left(d_{2 \theta, \lambda}-d_{h k l}+\delta_{2 \theta, \lambda}\right)^{2}}{\sigma_{2 \theta, \lambda}^{2}}\right]\right\}
\end{aligned}
$$

The parameters $\sigma$ and $\delta$ will depend on how the intersection of the profile function with the Bragg diffraction lines is defined, namely where $\Omega\left(d_{2 \theta, \lambda}-d_{h k l}\right)$ is normalized to

$$
\int_{-\infty}^{+\infty} \Omega\left(d_{2 \theta, \lambda}-d_{h k l}\right) \mathrm{d} d_{2 \theta, \lambda}=1 .
$$

For a common treatment of all Bragg reflections, we consider $\Omega$ along curves intersecting the Bragg lines $\lambda=2 d \sin \theta$ orthogonally for all values of $d$. These curves are obtained by solving the differential equation
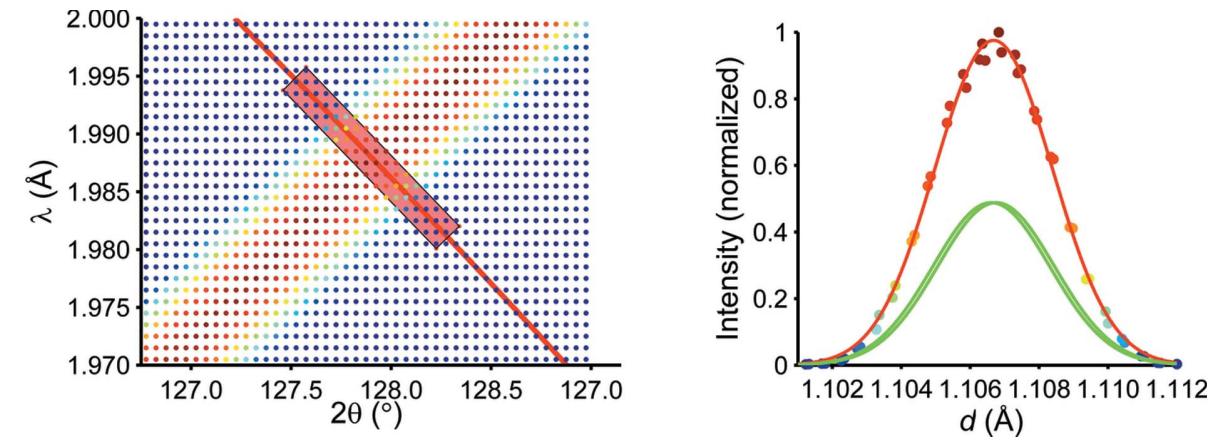

Data points contributing to a single slice indicated by the pale red rectangle (left) for which the orthogonal cut (red line) is taken at $2 \theta=127.8^{\circ}$ and $\lambda=1.988 \AA$, corresponding to a Bragg peak at $d=1.1067 \AA$. Intensity plotted against $d$ value of each data point (right) in which the red line is a fitted curve using the sum of two Gaussians and the green curves represent the single Gaussians. 


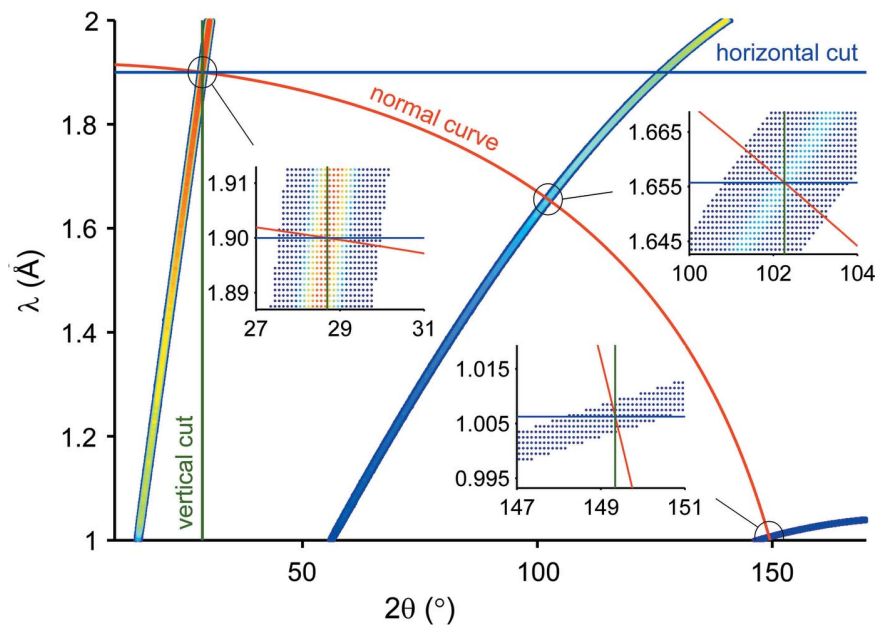

Figure 3

Course of the 100, 222 and 552 reflections and the resulting data of the VITESS simulation, together with lines for the horizontal, vertical and normal cuts. Insets show an enlarged version of the cross sections.

$$
\frac{\mathrm{d} \lambda}{\mathrm{d} \theta}=-\frac{1}{2 d \cos \theta},
$$

where $\lambda$ and $d$ have to be understood as dimensionless values. After expressing $d$ through $\lambda$ and $\theta$ according to the Bragg relation, we arrive at the expression

$$
d_{\perp}=\left(\lambda^{2}-2 \ln \cos \theta\right)^{1 / 2}
$$

Here $d_{\perp}(=\lambda$ at $\theta=0)$ is an alternative coordinate that together with $d$-gives a new orthogonal coordinate system.

It is obvious that considering orthogonal trajectories to the Bragg reflections is the most appropriate description for defining the profile function $\Omega$, and it exploits the twodimensional information most efficiently. This is illustrated in Fig. 3 where, taking the 100, 222 and 552 reflections of $\mathrm{Rh}_{0.81} \mathrm{Fe}_{3.19} \mathrm{~N}$ as examples, the horizontal and vertical cross sections are shown for comparison. Indeed, the horizontal cut corresponds to a monochromatic measurement and the vertical cut to a TOF measurement at a fixed angle. Additionally the inclination of the cuts orthogonal to the reflection changes over the diffraction pattern. For the 100 reflection the orthogonal cut is almost comparable to the horizontal cut,
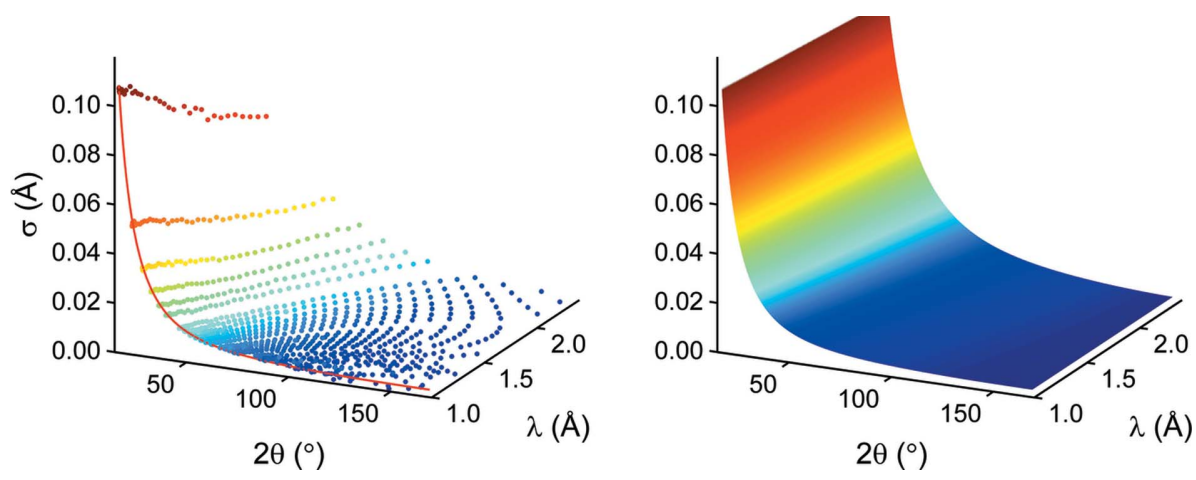

Figure 4

$\sigma$ values extracted at a large number of different points in the diffraction pattern (left) and fitted surface to the $\sigma$ values (right) with $R^{2}=0.993$. The red line connects data points of constant wavelength. while for the 552 reflection we almost have a vertical cut. For the 222 reflection the orthogonal cut lies in between.

It is preferable to separate the instrumental contribution to the peak shape and width from additional sample effects such as strain, stress or size effects. In a real experiment, one would use standard samples to determine such parameters. In the present modeling study we will simply use our simulated and idealized sample. Extracting the $\sigma$ and $\delta$ values at various points of the diffraction pattern leads to the distributions shown in Fig. 4. Similar to the Caglioti formula (Caglioti et al., 1958) we find an appropriate analytical description of the instrument characteristics using the parameters $\sigma$ and $\delta$ as a function of $2 \theta$ and $\lambda$ :

$$
\begin{aligned}
& \sigma_{2 \theta, \lambda}=d\left\{\left[\left(u_{1} \lambda+u_{2}\right) \cot \theta\right]^{2}+\left(\frac{u_{3} h}{\lambda m_{\mathrm{n}} L}\right)^{2}\right\}^{1 / 2}, \\
& \delta_{2 \theta, \lambda}=d\left\{\left[\left(v_{1} \lambda+v_{2}\right) \cot \theta\right]^{2}+\left(\frac{v_{3} h}{\lambda m_{\mathrm{n}} L}\right)^{2}\right\}^{1 / 2},
\end{aligned}
$$

with $u_{1}$ and $u_{2}$ describing a wavelength-dependent angular resolution $(\Delta \theta)$, and $u_{3}$ essentially representing the time resolution $(\Delta t)$ of the resolution function. The parameters $v_{1}$, $v_{2}$ and $v_{3}$ are coefficients of the displacement function, and $L$ is the distance from the chopper to the detector at position $2 \theta$ Furthermore, $h$ and $m_{\mathrm{n}}$ represent Planck's constant and the neutron mass, respectively. The refinement results of the profile parameters were obtained from a sufficiently large number of orthogonal cuts. The variations of $\sigma$ and $\delta$ versus $2 \theta$ are similar, and both are smooth functions, as expected. It seems that there are minor deviations around $2 \theta=45$ and $135^{\circ}$ coinciding with the corners of the cylindrical detector, thereby corresponding to a discontinuity in the derivative of $\Omega$. It is straightforward, however, to define $\Omega$ in a piece-wise fashion and also separately for the three detector elements. This has not been done here, because we consider these effects as negligible at the current stage. The fit of the parameters $u_{1-3}$ and $v_{1-3}$ nicely matches the measured $\sigma$ and $\delta$ values and yields an overall $R^{2}$ value of 0.993 (see Fig. 4).

When replacing $\sigma$ and $\delta$ in equation (2) with their corresponding analytical functions, one may fit the measured data using the resulting profile function (which now depends on $2 \theta$ and $\lambda$ ) in equation (1), just like in typical 'structural' Rietveld refinements carried out up to the present day. The background $b$ as originating from the incoherent scattering is accounted for by a single parameter. In a first attempt at structural refinement, only a limited number of parameters were refined, namely the scale $S$ and background $b$ as well as the lattice parameters, while keeping the internal structural parameters (atomic site, displacement parameter etc.) fixed. The results of the 
pattern fitting for the $\mathrm{Rh}_{0.81} \mathrm{Fe}_{3.19} \mathrm{~N}$ and $\mathrm{CuNCN}$ phases are summarized in Table 3. Fig. 5 presents the simulated diffraction pattern, the calculated diffraction pattern and their difference ( $\left.I_{\text {calculated }}-I_{\text {simulated }}\right)$. The overall agreement seems to be satisfactory. Nonetheless, small deviations of the calculated pattern from the simulated data, especially at high $2 \theta$ angles and high $\lambda$ values, can be observed. At high $\lambda$ values the intensity in the simulated diffraction patterns falls off near the maximum value of $2.4 \AA$, while in the calculated pattern the intensity remains unchanged. One may note that the rather sharp diffraction lines in backscattering were imperfectly described by the too coarse binning. Of course, a finer binning is always possible for an appropriate refinement of the analytical profile description, and with the choice to limit additional computational effort to the relevant backscattering region.

By neglecting any sample effects aside from the scattering process, the obtained parameterized profile function as
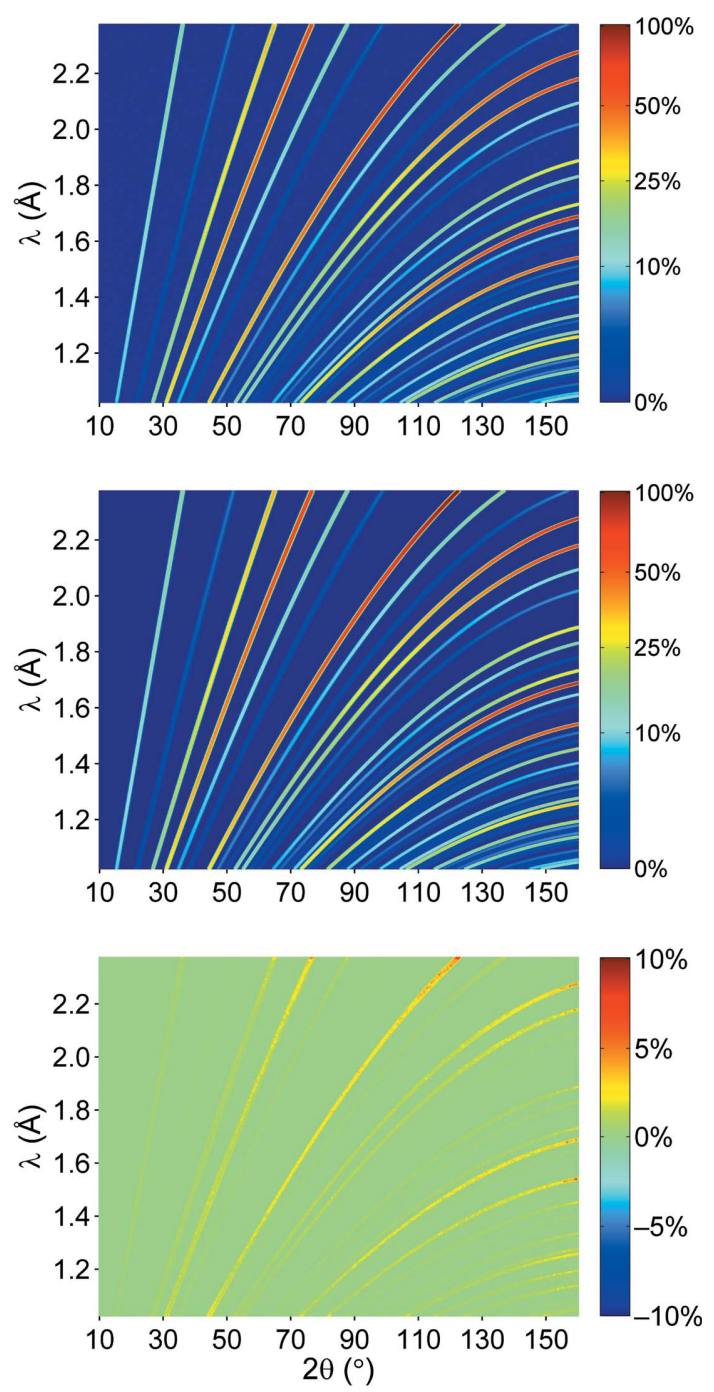

Figure 5

Simulated (top), calculated (middle) and differential (bottom) diffraction pattern of $\mathrm{Rh}_{0.81} \mathrm{Fe}_{3.19} \mathrm{~N}$ at POWTEX. The color bar of each picture denotes the intensity as a percentage of the largest intensity peak in the simulated diffraction pattern.
Table 3

Results of the pattern fitting for the simulated data of $\mathrm{Rh}_{0.81} \mathrm{Fe}_{3.19} \mathrm{~N}$ and CuNCN.

\begin{tabular}{lll}
\hline & $\mathrm{Rh}_{0.81} \mathrm{Fe}_{3.19} \mathrm{~N}$ & $\mathrm{CuNCN}$ \\
\hline No. parameters & 3 & 5 \\
No. data points & 2.16 million & 2.16 million \\
No. reflections & 68 & 389 \\
Calculation time $(\mathrm{min}) \dagger$ & $<2$ & $\simeq 30$ \\
Scale & $2.435(1) \times 10^{-3}$ & $2.664(1) \times 10^{-4}$ \\
Background & $1.79(7) \times 10^{-4}$ & $1.96(2) \times 10^{-4}$ \\
Lattice parameters $(\mathrm{A})$ & $a=3.83364(1)$ & $a=2.98905(1), b=6.14192(2)$, \\
& & $c=9.40087(2)$ \\
$R_{\mathrm{p}}$ & 0.060 & 0.050 \\
\hline
\end{tabular}

$\dagger$ ASUS K73S Notebook with Intel Core i5-2410M (2 Cores @ 2.3 GHz) and 6 GB of RAM.

resulting from the $\mathrm{Rh}_{0.81} \mathrm{Fe}_{3.19} \mathrm{~N}$ simulation should be regarded as the instrumental profile function, which does not depend on the actual sample. Therefore, the profile function is the valid base for fitting the diffraction patterns of standard samples without discernible size/strain contributions. This is exemplified by the Rietveld refinement of simulated CuNCN
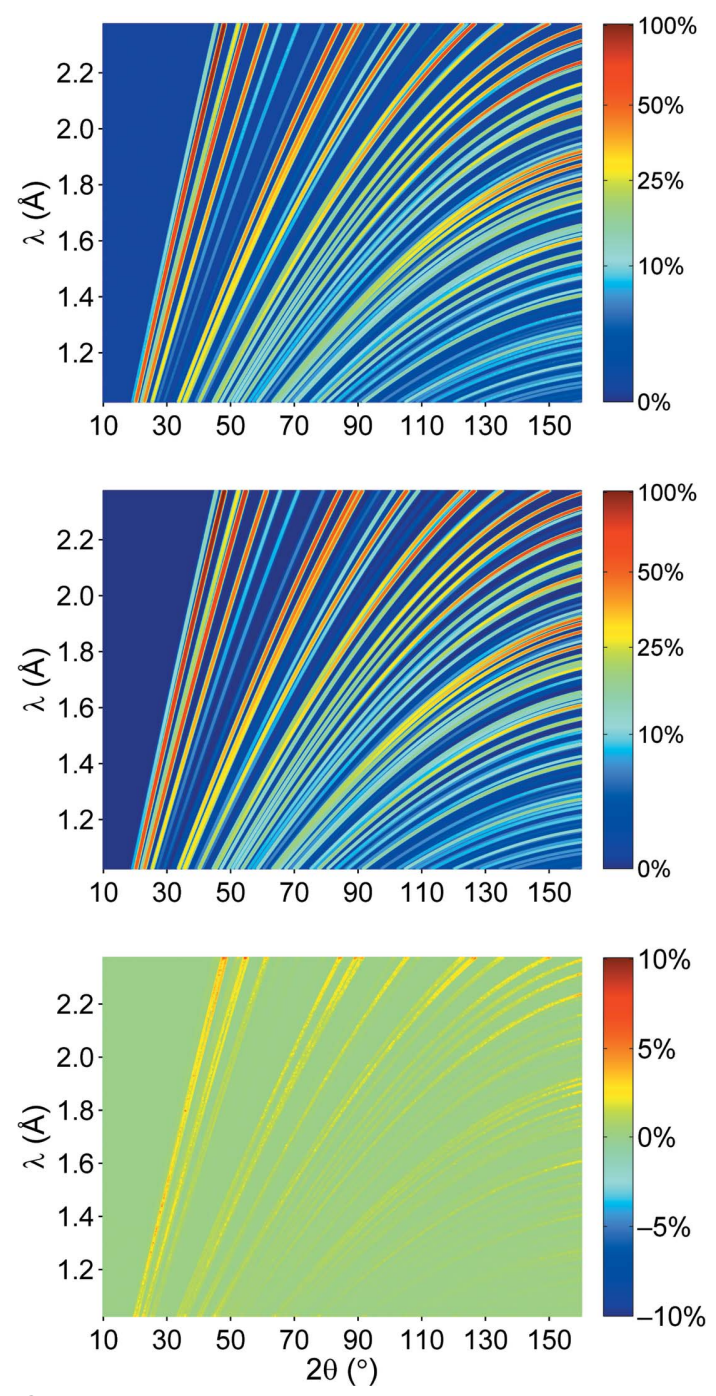

Figure 6

Same as in Fig. 5 but for CuNCN. 
Table 4

Results of the two-dimensional pattern fitting for the experimental data of CuNCN (POWGEN) in comparison to the FullProf refinement.

\begin{tabular}{lll}
\hline & $\begin{array}{l}\text { CuNCN (two- } \\
\text { dimensional Rietveld) }\end{array}$ & $\begin{array}{l}\text { CuNCN } \\
\text { (focused) }\end{array}$ \\
\hline No. parameters & 5 & 27 \\
No. data points & 0.62 million & 5920 \\
No. reflections & 515 & 276 \\
Calculation time (min) $\dagger$ & 60 & $\simeq 1$ \\
Scale & $0.0160(1)$ & $1.021(6)$ \\
Background & Single value of & Interpolation between \\
& $0.840(3)$ & 30 selected points \\
Lattice parameters $(\AA)$ & $a=2.98920(7)$, & $a=2.98908(8)$, \\
& $b=6.1423(2)$, & $b=6.1420(3)$, \\
$R_{\mathrm{p}}$ & $c=9.4012(3)$ & $c=9.4009(4)$ \\
\hline
\end{tabular}

$\dagger$ ASUS K73S Notebook with Intel Core i5-2410M (2 Cores@2.3 GHz) and 6 GB of RAM.

data but using the identical parameterization of the profile function as in the $\mathrm{Rh}_{0.81} \mathrm{Fe}_{3.19} \mathrm{~N}$ case. The diffraction pattern is shown in Fig. 6. Note that even regions with severe peak overlap are very well described, and the calculated intensities nicely match the simulated ones over almost the entire diffraction pattern. The results of the pattern fitting are summarized in Table 3. Minor deviations are only observed at large diffraction angles and wavelength, as mentioned above, but otherwise the agreement is very good.

As noted by an insightful reviewer, the aforementioned strategy - refining against a simulated, Monte Carlo derived data set by a novel two-dimensional Rietveld method and gauging the quality of the latter only by comparing simulated and theoretical intensities - might look questionable because experimental data are totally lacking; we reiterate, however, that there are no experimental data since the POWTEX machine is still under construction. Nonetheless, it is possible to experimentally test the novel method although real data must then come from a different source. To do so and follow the strategies laid out in the preceding part, we have tested our approach using a real data set for $\mathrm{CuNCN}$ which was obtained at the POWGEN instrument. Because of the natural wavelength dependence of the moderator pulse and the heartshaped detector arrangement of POWGEN, the instrument
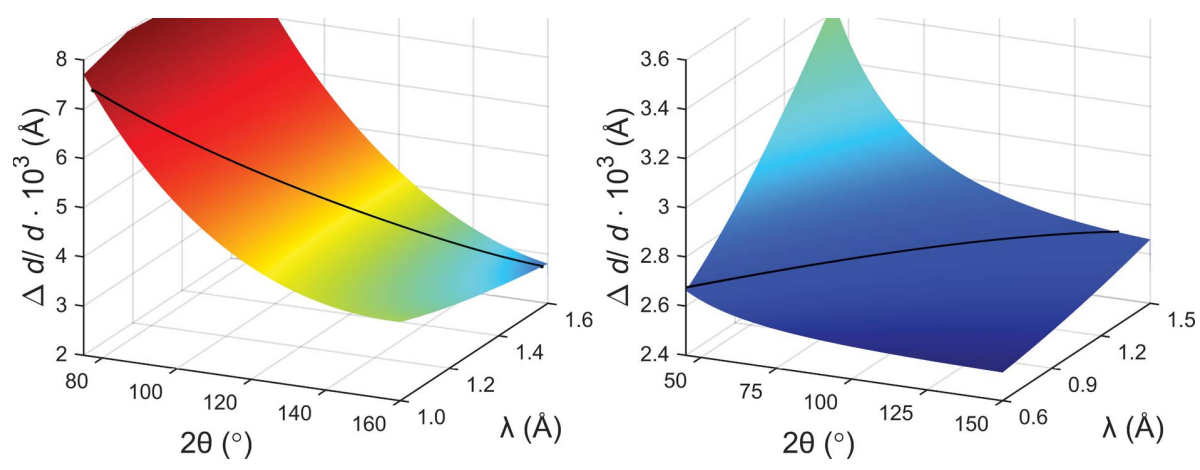

Figure 7

Comparison of resolution functions for POWTEX (left) and POWGEN (right). The black curve is a visualization of points belonging to a single reflection with $d=0.8 \AA$. yields almost constant resolution $\Delta d / d$, while a varying resolution function is more appropriate for the required use of pulse-shaping choppers at the POWTEX instrument.

The flexibility of our new approach which includes a varying resolution function also has an important benefit for the instrument design, simply because the detector shape is much simpler and more economical in terms of construction costs, i.e. cylindrical and covering a large solid angle. The profile function is mainly determined by the FWHM of each reflection independent of the actual $2 \theta$ and $\lambda$ value of the data point. This is depicted in Fig. 7, where the resolution function for the POWTEX instrument (left) is compared with that of the POWGEN instrument (right). For an arbitrarily chosen reflection at $d=0.8 \AA$ (black line) it is obvious that POWTEX's resolution changes with $2 \theta$ and $\lambda$ while for the POWGEN instrument it essentially remains the same. In particular for the CuNCN measurement, we note that the sample exhibits microstrain discernible by the FWHM of each reflection, an effect which had to be corrected by applying a strain correction $\left(\mathrm{DST}^{2}\right)$ in the quartic form for Laue class mmm (Stephens, 1999).

The FWHM of each reflection has thus been handled according to the notation used in FullProf (http://www.ill.eu/ sites/fullprof/) leading to

$$
\sigma_{h k l}=\left[\operatorname{Sig} 0^{2}+\left(\operatorname{Sig} 1^{2}+\operatorname{DST}^{2}\right) d_{h k l}^{2}+\operatorname{Sig} 2^{2} d_{h k l}^{4}\right]^{1 / 2} .
$$

Sig0, Sig1 and Sig2 are refinable parameters defining the halfwidth of the peak at value $d_{h k l}$ and DST defines an additional contribution by microstrain. The measured diffraction data and the results from refinement are shown in Fig. 8. Compared to conventional one-dimensional diffraction patterns, the statistical variation is of course more apparent in the twodimensional distribution of the data which were measured in $7.5 \mathrm{~h}$. The two-dimensional refinement yields an excellent data description as seen from the difference map in Fig. 8. The refinement parameters are given in Table 4 and indicate excellent agreement with the parameters and accuracies obtained by the standard Rietveld (FullProf) refinement; as expected, however, the residual value for the two-dimensional refinement is larger, thereby reflecting the much lower statistical significance per data pixel. The comparison also reveals that the conventional analysis should be typically fine for the analysis of POWGEN data which goes back to the instrument optimization with respect to resolution properties. The novel approach, however, additionally offers a more thorough check of the data quality of TOF diffractometers at current spallation sources. For an instrument like POWTEX and, likewise, for future powder diffractometers at the ESS (European Spallation Source) which will also use pulse-shaping choppers, the new approach will fully exploit the best 

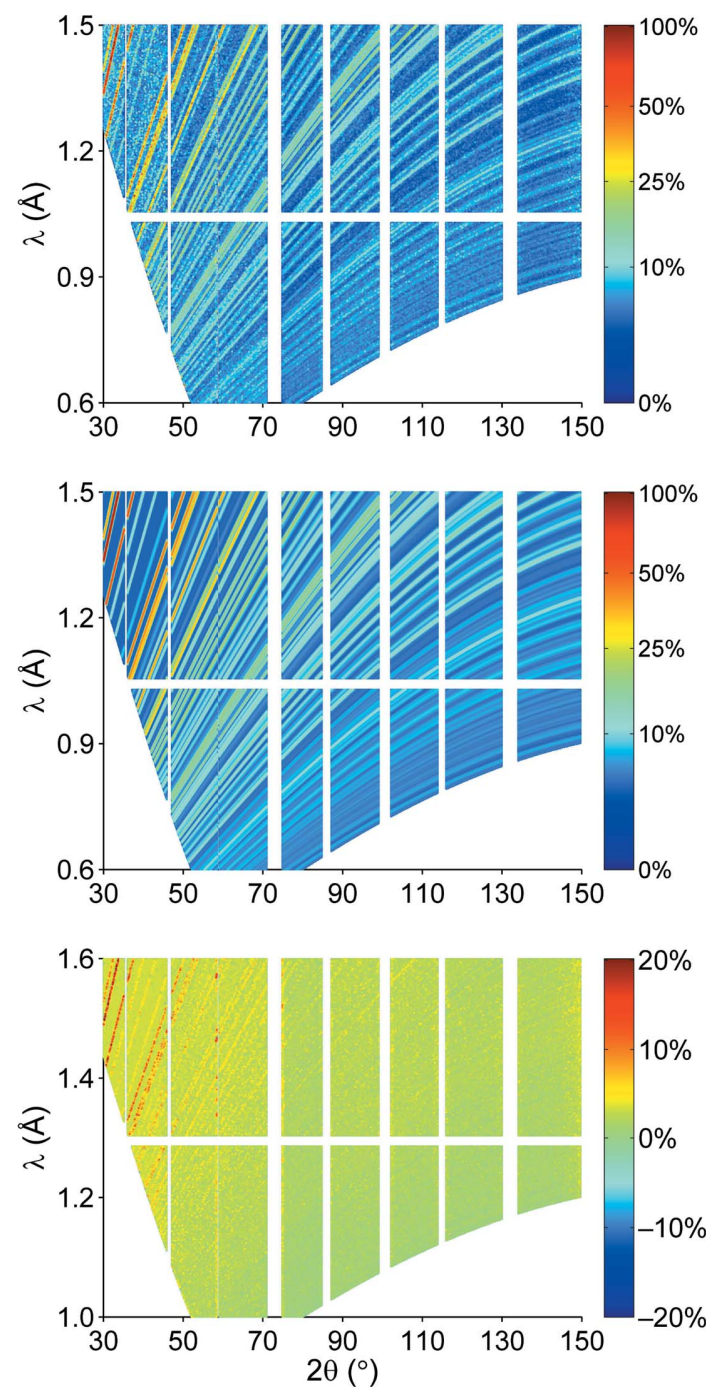

Figure 8

Measured (top), fitted (middle) and differential (bottom) diffraction pattern of CuNCN data from POWGEN. The color bar of each picture denotes the intensity as a percentage of the largest intensity peak in the simulated diffraction pattern.

resolution properties which would be lost by averaging for today's standard refinement procedures.

Since the POWTEX instrument reflects, by its very design, a user-driven approach of the solid-state chemistry community to structural characterization, the developers ought to carefully react to gathered user feedback. One main concern of today's users might be foreseeable in the unfamiliar twodimensional diffraction pattern (Fig. 1) and especially the data comparison within the novel Rietveld strategy. We therefore point out that it is quite easy to generate all sorts of reduced plots, such as the traditional 'Rietveld pattern' of intensity versus diffraction angle, irrespective of the fact that the data treatment and refinement will be done with the full twodimensional data for reasons of superior refinement quality. A comparison with a standard one-dimensional FullProf refinement (Jacobs et al., 2013) is shown in Fig. 9. Furthermore, the integration of POWTEX's intensities to a one-dimensional pattern is also possible. In combination with further developed
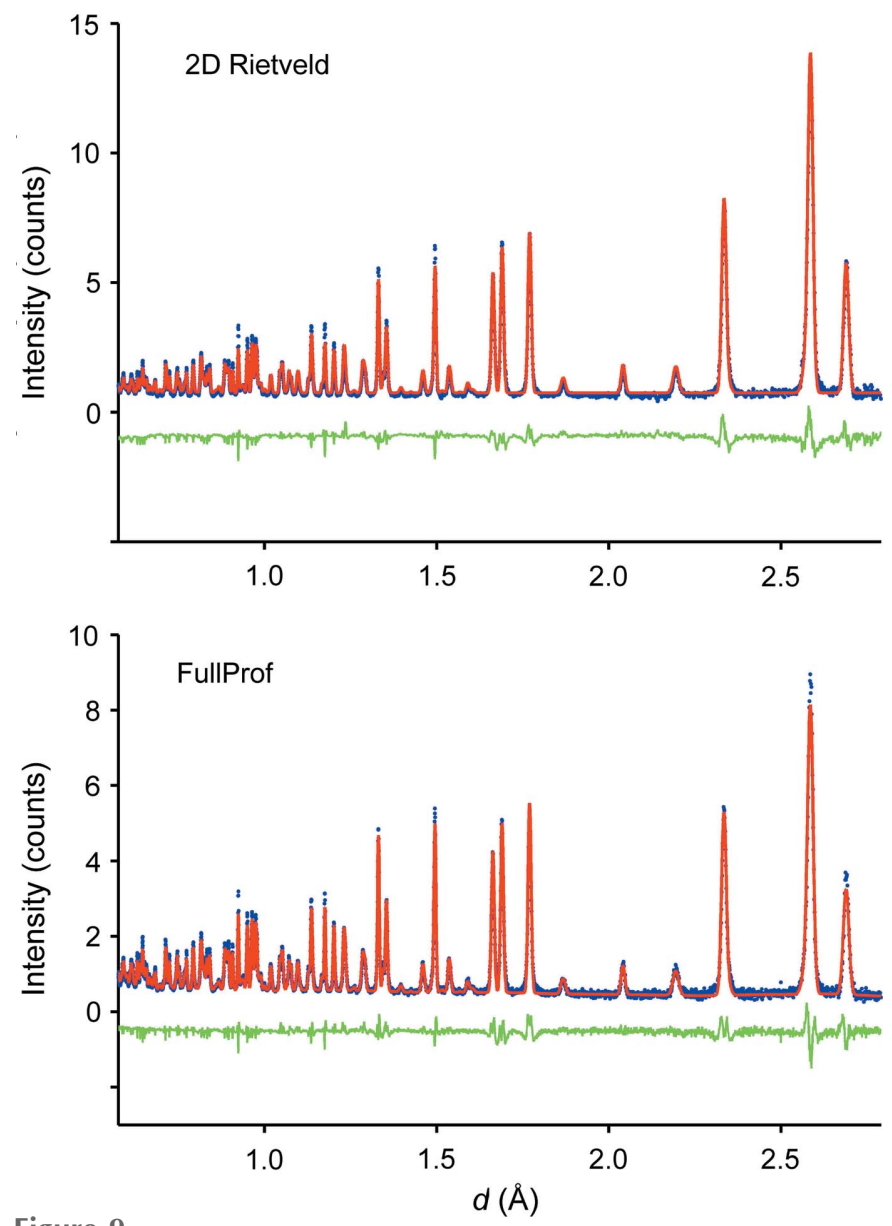

Figure 9

Top: conventional one-dimensional diffraction pattern of $\mathrm{CuNCN}$ derived from the two-dimensional Rietveld refinement. Bottom: comparison with the standard Rietveld refinement using FullProf.

techniques, e.g. nonlinear multi-bank approaches comparable to POLARIS or GEM (Hannon, 2005; Hull et al., 1992), one may at least partially overcome some of the above-mentioned issues and, as a benefit, adopt the data treatment more easily for POWTEX in contemporary refinement software. However, this is out of the scope of this article and was not elaborately tested.

\section{Conclusion and outlook}

We have demonstrated a simultaneous Rietveld refinement of angular- and wavelength-dispersive two-dimensional data sets. The latter were based on simulated Monte Carlo data using the layout of the POWTEX instrument which has been particularly optimized to benefit from a smooth but varying resolution with the highest resolution at large $2 \theta$.

In a first test we used simulated data obtained from the VITESS program package based on an idealized instrument layout and the structural models of $\mathrm{Rh}_{0.81} \mathrm{Fe}_{3.19} \mathrm{~N}$ and $\mathrm{CuNCN}$ as idealized samples. Similar to procedures on existing instruments, these 'standard' samples serve as input to determine the instrumental profile function as a function of $2 \theta$ and $\lambda$. Once established on the basis of real data, this instrumental 
profile function will be provided to the user. If deviations of the observed peak shape from the instrumental profile function should occur, these can then be attributed to sample effects and may be addressed accordingly.

In contrast to the POWTEX design, the TOF powder diffractometer POWGEN at the SNS in Oak Ridge, for example, has a deliberately chosen detector design which tries to minimize $\Delta d / d$ by best matching the angular contribution $\Delta \theta \cot \theta$ to the relative time resolution $\Delta t / t$. The latter is fairly independent of the wavelength owing to the moderators' natural moderation time at short-pulse spallation sources while, as in our case, pulse shaping results in a constant absolute time resolution $\Delta t$. Because POWGEN has been designed in such a way that the resolution in time and angle are matched to each other, one may expect that it is possible to reduce their measured two-dimensional data to a onedimensional data set without severe compromises in quality. However, it is also straightforward to test the two-dimensional approach with experimental POWGEN data. For this second test, we used such an unreduced data set of a CuNCN sample measured at POWGEN and showed how to successfully apply our two-dimensional approach using these data; thus, the feasibility of the two-dimensional refinement method for experimental data has been validated. Experienced TOF diffraction users, who know that, sometimes, measured data are deliberately discarded to avoid accuracy loss introduced by data integration, might also appreciate the chance to check the data quality. Furthermore, we believe that the novel approach will have an impact not only on data analysis, since it allows more freedom to drive the instrumental design towards less complex detector arrangements and, most favorably, to the cylindrical POWTEX geometry with its axis along the beam direction. Therefore, the proposed approach seems to be of interest for instruments based on a similar geometry concept such as POLARIS at ISIS, Super-HRPD at JPARC and DREAM, which is designed for the ESS.

The distinct advantage of two-dimensional refinements with better control and analysis of the background makes a particularly interesting case for the parasitic incoherent scattering of hydrogen, which is typically inelastic and depends on the incident wavelength (Henry et al., 2009; Wilson et al., 2014). Such an effect should be rather obvious in two-dimensional data, in contrast to integrated one-dimensional data, and it should also be possible to model (for example, subtract) the phenomenon because of its known wavelength dependence.

With the fundamentals of two-dimensional data profiling laid out, future developments will aim at incorporating more sample effects (e.g. preferred orientation, absorption and such like). The given proof-of-concept will hopefully motivate the incorporation of this novel approach into existing programs such as FullProf, GSAS, MAUD, TOPAS or JANA, which will definitely be needed for future user applications.

\section{Acknowledgements}

Parts of this work are based upon experiments performed at the POWGEN instrument operated by JCNS at the Spallation
Neutron Source (Oak Ridge) and were thus sponsored by the Scientific User Facilities Division, Office of Basic Energy Sciences, US Department of Energy. The authors want to thank Olivier Gourdon and Ashfia Huq at the SNS for their kind help during the measurements on the CuNCN sample. The authors gratefully acknowledge the financial support provided by JCNS to perform the neutron scattering measurements at the Spallation Neutron Source, Oak Ridge, USA. We would also like to thank two perceptive reviewers for their suggestions and Joachim Wuttke for help in clarifying some aspects in the final draft. The financial support by the Federal Ministry of Research and Education (BMBF) for the POWTEX project (05K13PA1) is gratefully acknowledged.

\section{References}

Avdeev, M., Jorgensen, J., Short, S. \& Von Dreele, R. B. (2007). J. Appl. Cryst. 40, 710-715.

Bacon, G. E. \& Thewlis, J. (1949). Proc. R. Soc. London Ser. A, 196, 50-64.

Bragg, W. H. \& Bragg, W. L. (1913). Proc. R. Soc. London Ser. A, 88, 428-438.

Bruker (2005). TOPAS. Version 3. Bruker AXS, Karlsruhe, Germany.

Caglioti, G., Paoletti, A. \& Ricci, F. P. (1958). Nucl. Instrum. 3, 223 228.

Chapon, L. C., Manuel, P., Radaelli, P. G., Benson, C., Perrott, L., Ansell, S., Rhodes, N. J., Raspino, D., Duxbury, D., Spill, E. \& Norris, J. (2011). Neutron News, 22(2), 22-25.

Cheetham, A. K. \& Taylor, J. C. (1977). J. Solid State Chem. 21, $253-$ 275.

Conrad, H., Brückel, T., Schäfer, W. \& Voigt, J. (2008). J. Appl. Cryst. 41, 836-845.

Dinnebier, R. E. \& Billinge, S. J. L. (2008). Powder Diffraction: Theory and Practice. Cambridge: Royal Society of Chemistry.

Dollase, W. A. (1986). J. Appl. Cryst. 19, 267-272.

Elf, F., Gilles, R., Artus, G. R. J. \& Roth, S. (2002). Appl. Phys. Mater. Sci. Process. 74, s1477-s1479.

Finger, L. W., Cox, D. E. \& Jephcoat, A. P. (1994). J. Appl. Cryst. 27, 892-900.

Fischer, P., Frey, G., Koch, M., Könnecke, M., Pomjakushin, V., Schefer, J., Thut, R., Schlumpf, N., Bürge, R., Greuter, U., Bondt, S. \& Berruyer, E. (2000). Physica B, 276-278, 146-147.

Fischer, W. E. (1997). Physica B, 234-236, 1202-1208.

Garlea, V. O., Chakoumakos, B. C., Moore, S. A., Taylor, G. B., Chae, T., Maples, R. G., Riedel, R., Lynn, G. W. \& Selby, D. (2010). Appl. Phys. A, 99, 531-535.

Hannon, A. C. (2005). Nucl. Instrum. Methods Phys. Res. Sect. A, 551, 88-107.

Hansen, T. C., Henry, P. F., Fischer, H. E., Torregrossa, J. \& Convert, P. (2008). Meas. Sci. Technol. 19, 034001.

Henry, P. F., Weller, M. T. \& Wilson, C. C. (2009). J. Appl. Cryst. 42, 1176-1188.

Hoelzel, M., Senyshyn, A., Juenke, N., Boysen, H., Schmahl, W. \& Fuess, H. (2012). Nucl. Instrum. Methods Phys. Res. Sect. A, 667, 32-37.

Houben, A. P., Müller, P., von Appen, J., Lueken, H., Niewa, R. \& Dronskowski, R. (2005). Angew. Chem. Int. Ed. 44, 7212-7215.

Houben, A., Schweika, W., Brückel, T. \& Dronskowski, R. (2012). Nucl. Instrum. Methods Phys. Res. Sect. A, 680, 124-133.

Houben, A., Šepelák, V., Becker, K.-D. \& Dronskowski, R. (2009). Chem. Mater. 21, 784-788.

Howard, C. J. (1982). J. Appl. Cryst. 15, 615-620.

Hull, S., Smith, R. I., David, W. I. F., Hannon, A. C., Mayers, J. \& Cywinski, R. (1992). Physica B, 180-181, 1000-1002. 
Huq, A., Hodges, J. P., Gourdon, O. \& Heroux, L. (2011). EPDIC-12. Z. Kristallogr. Proc. 1, 127-135.

Ikeda, Y. (2005). Neutron News, 16(1), 20-24.

Jacobs, P., Houben, A., Tchougréeff, A. L. \& Dronskowski, R. (2013). J. Chem. Phys. 139, 224707.

Kamiyama, T., Oikawa, K., Tsuchiya, N., Osawa, M., Asano, H., Watanabe, N., Furusaka, M., Satoh, S., Fujikawa, I., Ishigaki, T. \& Izumi, F. (1995). Physica B, 213-214, 875-877.

Kuhs, W. F. \& Klein, H. (2008). Lecture at PD2DD Workshop, ILL, Grenoble, France.

Laar, B. van \& Yelon, W. B. (1984). J. Appl. Cryst. 17, 47-54.

Larson, A. C. \& Von Dreele, R. B. (1994). GSAS. Los Alamos National Laboratory, New Mexico, USA.

Lefmann, K. \& Nielsen, K. (1999). Neutron News, 10(3), 20-23.

Lengeler, H. (1998). Nucl. Instrum. Methods Phys. Res. Sect. B, 139, 82-90.

Lieutenant, K., Zendler, C., Manoshin, S., Fromme, M., Houben, A. \& Nekrassov, D. (2014). J. Neutron Res. 17, 45-51.

Lisowski, P. W. \& Schoenberg, K. F. (2006). Nucl. Instrum. Methods Phys. Res. Sect. A, 562, 910-914.

Liss, K.-D., Hunter, B., Hagen, M., Noakes, T. \& Kennedy, S. (2006). Physica B, 385-386, 1010-1012.

Liu, X., Wankeu, M. A., Lueken, H. \& Dronskowski, R. (2005). Z. Naturforsch. Teil B, 60, 593-596.

Lutterotti, L., Matthies, S. \& Wenk, H.-R. (1999). Proceedings of the 12th International Conference on Textures of Materials (ICOTOM12), Vol. 1, edited by J. A. Szpunar, p. 1599.

March, A. (1932). Z. Kristallogr. 81, 285-297.
Mason, T. E. et al. (2006). Physica B, 385-386, 955-960.

Modzel, G., Henske, M., Houben, A., Klein, M., Köhli, M., Lennert, P., Meven, M., Schmidt, C. J., Schmidt, U. \& Schweika, W. (2014). Nucl. Instrum. Methods Phys. Res. Sect. A, 743, 90-95.

Peters, J., Lieutenant, K., Clemens, D. \& Mezei, F. (2006). Z. Kristallogr. Suppl. pp. 189-194.

Petrríček, V., Dušek, M. \& Palatinus, L. (2006). Crystallogr. Comput. Syst. Inst. Phys. Acad. Sci. Czech. Repub. 162, 53.

Popa, N. C. (1998). J. Appl. Cryst. 31, 176-180.

Rietveld, H. M. (1969). J. Appl. Cryst. 2, 65-71.

Rodríguez-Carvajal, J. (1993). Physica B, 192, 55-69.

Rodriguez-Carvajal, J. (1997). FullProf2000. Version 3.2, pp. 20-22. Laboratoire Léon Brillouin, Saclay, France.

Schäfer, W., Jansen, E., Skowronek, R., Will, G., Knight, K. S. \& Finney, J. L. (1992). Nucl. Instrum. Methods Phys. Res. Sect. A, 317, 202-212.

Stephens, P. W. (1999). J. Appl. Cryst. 32, 281-289.

Többens, D. M., Stüsser, N., Knorr, K., Mayer, H. M. \& Lampert, G. (2001). Mater. Sci. Forum, 378-381, 288-293.

Von Dreele, R. B., Jorgensen, J. D. \& Windsor, C. G. (1982). J. Appl. Cryst. 15, 581-589.

Wechsler, D., Zsigmond, G., Streffer, F. \& Mezei, F. (2000). Neutron News, 11(4), 25-28.

Wilson, C. C., Henry, P. F., Schmidtmann, M., Ting, V. P., Williams, E. \& Weller, M. T. (2014). Cryst. Rev. 20, 162-206.

Young, R. A. \& Wiles, D. B. (1982). J. Appl. Cryst. 15, 430-438.

Zsigmond, G., Lieutenant, K. \& Mezei, F. (2006). Neutron News, 13(4), 11-14. 